\title{
Angiopoietin-2 promotes extracellular matrix degradation in human degenerative nucleus pulposus cells
}

\author{
KUN WANG $^{1 *}$, LIANG KANG ${ }^{1 *}$, WEI LIU ${ }^{2 *}$, YU SONG $^{1}$, XINGHUO WU ${ }^{1}$, YUKUN ZHANG $^{1}$,

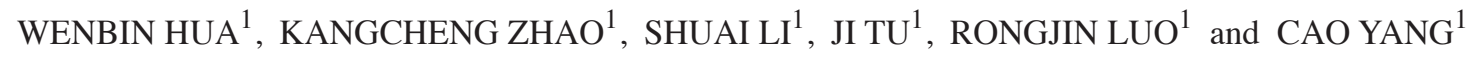 \\ ${ }^{1}$ Department of Orthopedics, Union Hospital, Tongji Medical College, Huazhong University of Science and Technology, \\ Wuhan 430022, China; ${ }^{2}$ Department of Orthopedics, First Hospital of Wuhan, Wuhan 430022, China
}

Received December 23, 2016; Accepted February 14, 2018

DOI: $10.3892 / \mathrm{ijmm} .2018 .3576$

\begin{abstract}
In contrast to healthy intervertebral discs (IVDs), degenerate IVDs become vascularized. Here, we determined the role of an angiogenesis promoter, angiopoietin (Ang)-2, in the pathology of IVD degeneration (IDD). We evaluated degree of IDD using the Pfirrmann grading system. We used quantitative real-time polymerase chain reaction and western blotting to analyze $A N G 2$ gene expression and Ang-2 protein levels, respectively. The involvement of Ang-2 in IVD degradation and regulation of nuclear factor- $\mathrm{\kappa B}$ (NF- $\mathrm{\kappa B})$ signaling was examined by immunohistochemistry, western blotting and immunofluorescence. As a result, 10 samples with grades II and III IDD were categorized as the mild IDD group; for comparison, another 10 specimens with grades IV and V constituted the severe IDD group. Ang-2 expression was significantly higher in severe IDD than in mild IDD. Exogenous Ang-2 administration led to increased production of catabolic proteinases and loss of aggrecan and collagen II in degenerative NP cell cultures, which was mediated by the NF-kB signaling pathway. Elevated Ang-2 levels also increased interleukin-1 $\beta$ expression in degenerative NP cells. We conclude that the release of Ang-2 aggravates NP cell degradation and plays an important role in IDD. Ang-2 may thus constitute a novel therapeutic target for the treatment of IVD.
\end{abstract}

\section{Introduction}

Low back pain (LBP) is a muscular disorder leading to severe social and economic burden. It has been shown that

Correspondence to: Professor Cao Yang, Department of Orthopedics, Union Hospital, Tongji Medical College, Huazhong University of Science and Technology, Wuhan 430022, China

E-mail: yangcao1971@sina.com

*Contributed equally

Key words: angiopoietin-2, intervertebral disc, degenerative nucleus pulposus cells, intervertebral disc degeneration, nuclear factor- $\kappa \mathrm{B}$
LBP is mainly associated with intervertebral disc (IVD) degeneration (IDD) $(1,2)$. IDD is characterized by a series of pathogenic processes, including cellular, biochemical, and structural impairment, which result in a metabolic imbalance of the extracellular matrix (ECM) $(3,4)$. Previous studies have demonstrated that catabolic proteinases, such as matrix metalloproteinases (MMPs) and a disintegrin and metalloproteinase with thrombospondin motifs (ADAMTSs), are upregulated in IDD; this is the main cause of ECM degradation during IDD progression $(5,6)$. While the proteolytic activity of MMPs and aggrecanases plays an important role in breaking down the disc matrix, another constant feature of disc degeneration is angiogenesis.

Angiopoietin (Ang) plays crucial roles in successful angiogenesis (7). Upregulation of Ang-2 expression constitutes a critical step in the initiation of the inflammatory process (8). On its own, Ang- 2 can recruit myeloid cells and induce inflammation even in the absence of preceding pro-inflammatory stimuli (9). Mice lacking Ang-2 cannot elicit an inflammatory response but administration of recombinant Ang-2 restores the inflammatory process (10). Ang-2 facilitates the activities of inflammatory molecules such as tumor necrosis factor and interleukin (IL)-1 exaggerates inflammatory responses $(11,12)$. These key inflammatory cytokines induce the expression of genes encoding matrix-degrading enzymes in IDD (13). Previous studies have shown that Ang-1 is crucial for the survival of nucleus pulposus (NP) cells (14). However, limited studies have explored the function of Ang-2 in degenerative NP cells.

Here, we investigated the role of Ang- 2 in human degenerative NP cells and IDD pathology. We verified the expression of Ang-2 in human degenerative NP cells and determined that its level increase with progressing degeneration. We demonstrated that Ang-2 participates in ECM degradation in degenerative NP cells, as well as in the occurrence and development of IDD. This effect is mediated by the nuclear factor- $\mathrm{KB}(\mathrm{NF}-\kappa \mathrm{B})$ signaling pathway. Ang-2 stimulated expression of IL- $1 \beta$ in human degenerative NP cells.

\section{Materials and methods}

The collection and staging of human NP tissue samples. This study was approved by the Institutional Ethics Review Board 
of Tongji Medical College of Huazhong University of Science and Technology. The investigation was carried out following the rules of the Declaration of Helsinki. The patients or their parents (on behalf of children) provided informed consent prior to the study. NP tissues were collected from patients $(n=20)$ undergoing spinal surgery. Patients undergoing surgery for burst fracture $(n=3)$, lumbar disc herniation $(n=8)$, lumbar spinal stenosis $(n=5)$ or spondylolysis $(n=4)$ were enrolled in this study. The patients comprised 23 males and 17 females aged from 13 to 59 years. The T2 weighted magnetic resonance images were obtained at 1.5 Tesla resolution and the tissues were evaluated using the Thompson grading system and Pfirrmann classification system $(15,16)$. Among the NP specimens, 3 specimens were classified as grade II and 7 specimens as grade III; 5 disc specimens from patients with lumbar disc herniation or spondylolysis were classified as grade IV and the others were grade $\mathrm{V}$.

Immunohistochemistry. Samples from human NP tissues were fixed in $4 \%$ paraformaldehyde in phosphate buffer $(\mathrm{pH} 7.4)$ for $24 \mathrm{~h}$. Paraffin sections were cut into $4-\mu \mathrm{m}$ thick sections. The sections were dewaxed in xylene, then rehydrated in graded alcohol series. Antigen retrieval was performed by microwave in Tris-EDTA pH 9.0 for $15 \mathrm{~min}$. Followed by washing with phosphate-buffered saline (PBS), the sections were blocked with endogenous peroxidase activities in $3 \% \mathrm{H}_{2} \mathrm{O}_{2}$. Subsequently the samples were incubated with anti-Ang-2 (sc-7015, 1:200; Santa Cruz Biotechnology, Inc., Santa Cruz, CA, USA), anti-collagen II (ab34712, 1:200) and anti-MMP13 (ab39012, 1:200) (both from Abcam, Cambridge, MA, USA). After labeling with a secondary antibody $(1: 2,000)$ conjugated to horseradish peroxidase, the sections were stained with diaminobenzidine (DAB) and counterstained with hematoxylin. Images were captured using an IX71 phase microscope (Olympus, Tokyo, Japan).

Isolation of NP cells. NP cells were isolated as previously described (17,18). After isolation, the NP cells were plated and expanded for 3 weeks in Dulbecco's modified Eagle's medium (DMEM) supplemented with $20 \%$ fetal bovine serum (FBS), and $1 \%$ penicillin/streptomycin at $37^{\circ} \mathrm{C}$ in a $5 \% \mathrm{CO}_{2}$ incubator. The culture medium was replaced twice a week. All NP cells were isolated from degenerated samples. The cells passaged two to three times were used for subsequent experiments.

Exogenous Ang-2 treatment of NP cells. When cultured NP cells reached $80 \%$ confluence, $2 \times 10^{4}$ cells were seeded into 96-well plates and subjected to serum starvation overnight to synchronize the cell cycles. The cells were then treated with various concentrations of exogenous Ang-2 (R\&D Systems, Minneapolis, MN, USA) for $24 \mathrm{~h}$. They were then harvested for mRNA and protein analysis. To evaluate the signaling pathways in NP cells, cells were treated with various concentrations of Ang-2 for $24 \mathrm{~h}$, and indicated concentrations of the NF- $\mathrm{BB}$ inhibitor BAY11-7082 (Beyotime, Nantong, China) were added for $1 \mathrm{~h}$. Each treatment was performed in 3 different wells.

Quantitative western blotting. The culture supernatants were collected and cells were lysed for $20 \mathrm{~min}$ in cold radioimmunoprecipitation (RIPA) lysis buffer (Beyotime, Beijing, China). Protein lysates were resolved by SDS-PAGE, and transferred onto polyvinylidene fluoride membranes (Amersham Biosciences, Piscataway, NJ, USA). The membranes were incubated with primary antibodies. In another experiment, nuclear protein was extracted using the CelLytic NuClear extraction kit (Sigma-Aldrich, St. Louis, MO, USA) following the manufacturer's instructions. Nuclear proteins were incubated with anti-p65 antibodies for $2 \mathrm{~h}$. Immunoreactive bands were quantified using ImageQuant LAS 400 software (GE Healthcare Life Sciences, Logan, UT, USA) and calculated by normalization to the reference bands of $\beta$-actin or lamin A. The primary antibodies used were as follows: anti-Ang-2 (sc-7015, 1:2,000) was purchased from Santa Cruz Biotechnology, Inc. Anti-collagen II (ab34712, 1:2,000), anti-aggrecan (ab3778, 1:1,500), anti-MMP13 (ab39012, 1:3,000), anti-ADAMTS4 (ab185722, 1:3,000), IL-1 $\beta$ (ab2105, 1:3,000) and anti-p65 antibodies (ab16502, 1:2,000) were all obtained from Abcam.

RNA extraction and $q R T-P C R$. Total RNA was isolated from harvested cells and tissues using TRIzol reagent (Invitrogen, Carlsbad, CA, USA) according to the manufacturer's instructions. qRT-PCR was carried out using the Power SYBR-Green PCR Master Mix and a 7900 HT thermocycler (Applied Biosystems, Foster City, CA, USA). Briefly, RNA was denatured for $5 \mathrm{~min}$ at $70^{\circ} \mathrm{C}$ and placed on ice for $5 \mathrm{~min}$. Denatured RNA was added to a mixture of MMLV-RT, MMLV-RT buffer, HRP (RRI)/RNase inhibitor, and dNTPs, and incubated for $60 \mathrm{~min}$ at $42^{\circ} \mathrm{C}$. The reagent was then inactivated by heating at $95^{\circ} \mathrm{C}$ for $5 \mathrm{~min}$. After amplification, relative gene expression levels were determined using the $2^{-\Delta \Delta \mathrm{Ct}}$ method. $\beta$-actin was used as an endogenous control to normalize mRNA levels. The expression of the following genes was investigated: Ang-2 forward, 5'-ACTGTGTCCTCTTCCA CCAC-3' and reverse, 5'-GGATGTTTAGGGTCTTGCTTT-3'; MMP13 forward, 5'-CCCAACCCTAAACATCCAA-3' and reverse, 5'-AAACAGCTCCGCATCAACC-3'; ADAMTS4 forward, 5'-ACCCAAGCATCCGCAATC-3' and reverse, 5'-TGCCCACATCAGCCATAC-3'; collagen II forward, 5'-TCCAGATGACCTTCCTACGC-3' and reverse, 5'-GGTAT GTTTCGTGCAGCCAT-3'; aggrecan forward, 5'-TGAGCGG CAGCACTTTGAC-3' and reverse, 5'-TGAGTACAGGAGGC TTGAG-3'; IL-1 $\beta$ forward, 5'-ATGGCTTATTACAGTG GCA-3' and reverse, 5'-TGTAGTGGTGGTCGGAGA-3', Homo $\beta$-actin forward, 5'-AGCGAGCATCCCCCAAAGTT-3' and reverse, 5'-GGGCACGAAGGCTCATCATT-3'.

Statistical analysis. All results are representative of three independent experiments, with three replicates per experiment, and expressed as the mean \pm SD. Two-tailed unpaired Student's t-test was used to compare two groups, and one-way analysis of variance (ANOVA) was used to compare three or more groups to test significant differences. $\mathrm{P}<0.05$ was considered to be statistically significant.

\section{Results}

Expression of Ang-2 in human NP tissues. To explore the role of Ang-2 in the pathogenesis of IDD, we first assessed 

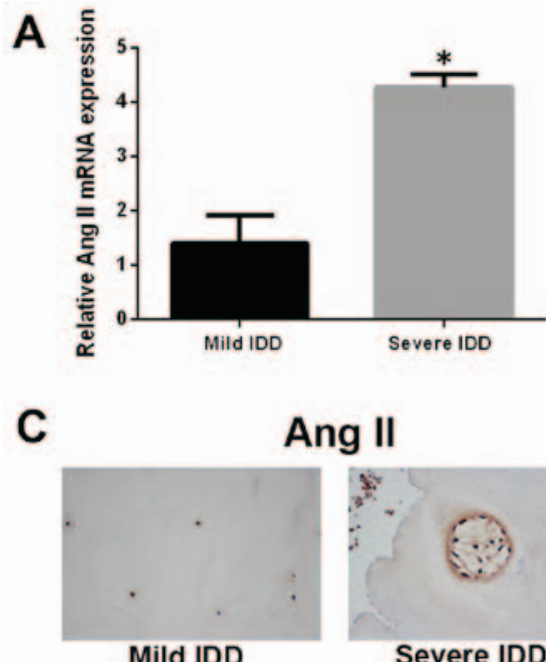

Ang II
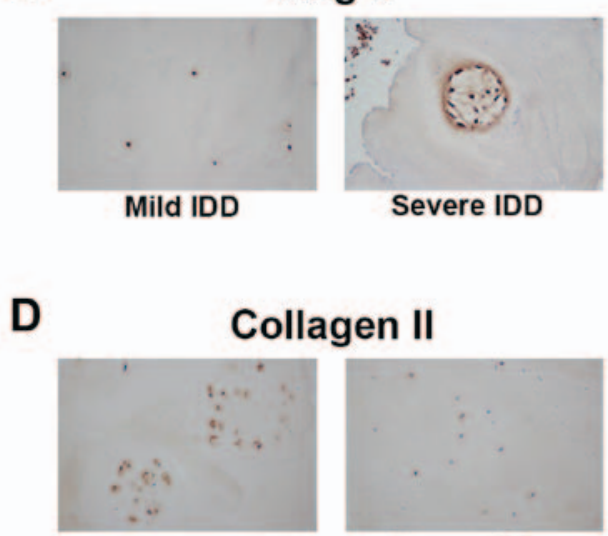

Mild IDD

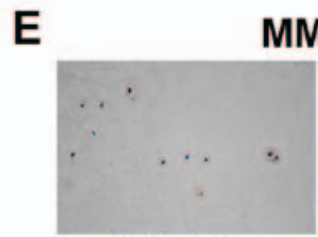

Mild IDD
MMP13

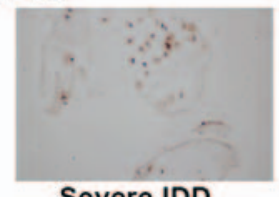

B
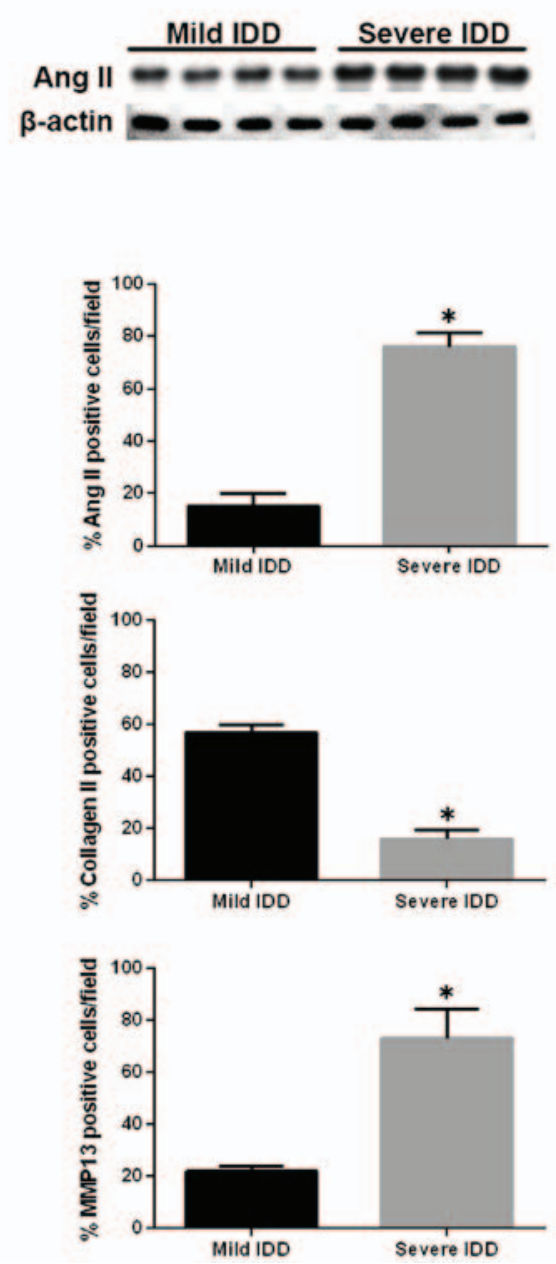

Figure 1. The expression of angiopoietin-2 (Ang-2) in human nucleus pulposus (NP) tissues. (A) Ang-2 mRNA and (B) protein levels in NP tissues from mild intervertebral disc degeneration (IDD) $(n=10)$ and severe IDD $(n=10)$ patients were analyzed by quantitative real-time polymerase chain reaction (qRT-PCR) and western blotting, respectively. $\beta$-actin was used as an internal control. Representative images of (C) Ang-2, (D) type II collagen and (E) MMP13 protein levelsin NP tissue $(n=20)$. The proteins were detected by immunohistochemistry. Magnification, $x 400$ *P<0.05 vs. mild IDD group.

the expression of the $A N G 2$ gene in human IVD samples by quantitative real-time polymerase chain reaction (qRT-PCR). The expression of $A N G 2$ gene was markedly upregulated in NP tissues from severe IDD samples compared with those from mild IDD samples (Fig. 1A). Similar results were observed by western blotting, and Ang-2 protein levels were significantly increased in the severe IDD group compared with the mild IDD group (Fig. 1B). Immunohistochemistry revealed that Ang-2-positive cells were significantly more numerous in the severe IDD group compared with the mild IDD group (Fig. 1C). Moreover, the amounts of type II collagen-positive cells and MMP13-positive cells were decreased and increased, respectively, in severe IDD compared with mild IDD group (Fig. 1D and E).

Ang-2 treatment promotes ECM degradation in human degenerative NP cells. We then investigated the impact of Ang-2 on ECM metabolism in degenerative NP cells. We measured the mRNA and protein levels of type II collagen and aggrecan by qRT-PCR and western blotting, respectively, in degenerative NP cells exposed to exogenous Ang-2. Ang-2 treatment resulted in a pronounced downregulation of type II collagen and aggrecan at both mRNA and protein levels (Fig. 2A-D). ECM catabolic proteinases, such as MMPs and ADAMTSs, are highly expressed in degenerative IVD tissue and cells, and are involved in ECM degradation during the development of IDD (19). We therefore measured the expression of MMP13 and ADAMTS4 in degenerative NP cells. We observed that the expression of MMP13 and ADAMTS4 on mRNA and protein levels was significantly increased in degenerative NP cells treated with exogenous Ang-2 compared with untreated controls (Fig. 2E-H).

Ang-2 induces activation of the $N F-\kappa B$ pathway. It has been reported that the NF- $\kappa \mathrm{B}$ pathway is aberrantly activated in IDD $(20,21)$. We therefore asked whether the NF- $\mathrm{B}$ signaling pathway is involved in Ang-2-induced ECM degradation. We analyzed $\mathrm{NF}-\kappa \mathrm{B}$ nuclear translocation by western blotting and immunofluorescence. As shown in Fig. 3A, Ang-2 treatment markedly increased the protein levels of NF- $\mathrm{B}$ p 65 in a nuclear extract, in a dose-dependent manner, which was indicative of the activation of the $N F-\kappa B$ pathway. The 


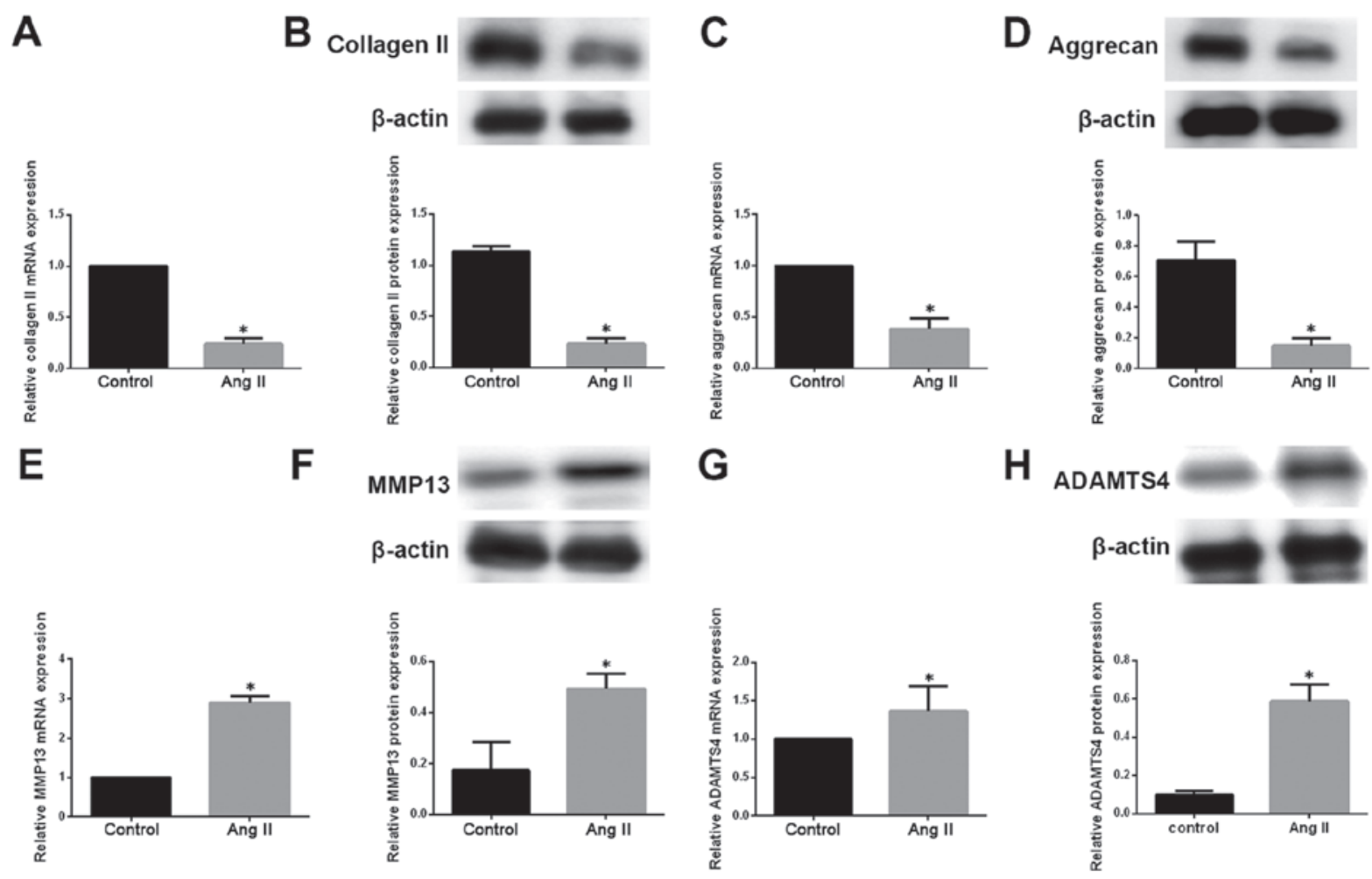

Figure 2. The effect of angiopoietin-2 (Ang-2) on extracellular matrix (ECM) degradation in human nucleus pulposus (NP) cells. NP cells were treated with exogenous Ang-2 $(10 \mu \mathrm{M})$ for $24 \mathrm{~h}$, with untreated cells serving as a control. (A and B) The mRNA and protein levels of type II collagen and (C and D) aggrecan in NP cells were analyzed by quantitative real-time polymerase chain reaction (qRT-PCR) and western blotting, respectively. (E and F) MMP13 and (G and H) ADAMTS4 mRNA and protein levels in NP cells, as determined by qRT-PCR and western blotting, respectively. $\beta$-actin was used as an internal control. ${ }^{*} \mathrm{P}<0.05$ vs. control group.

A

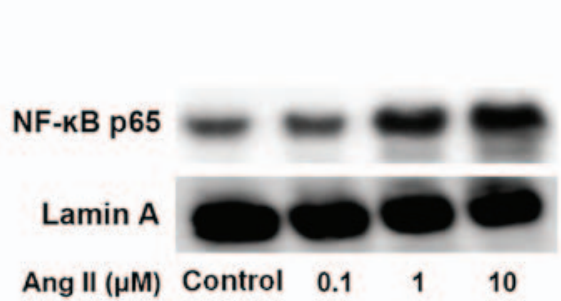

B
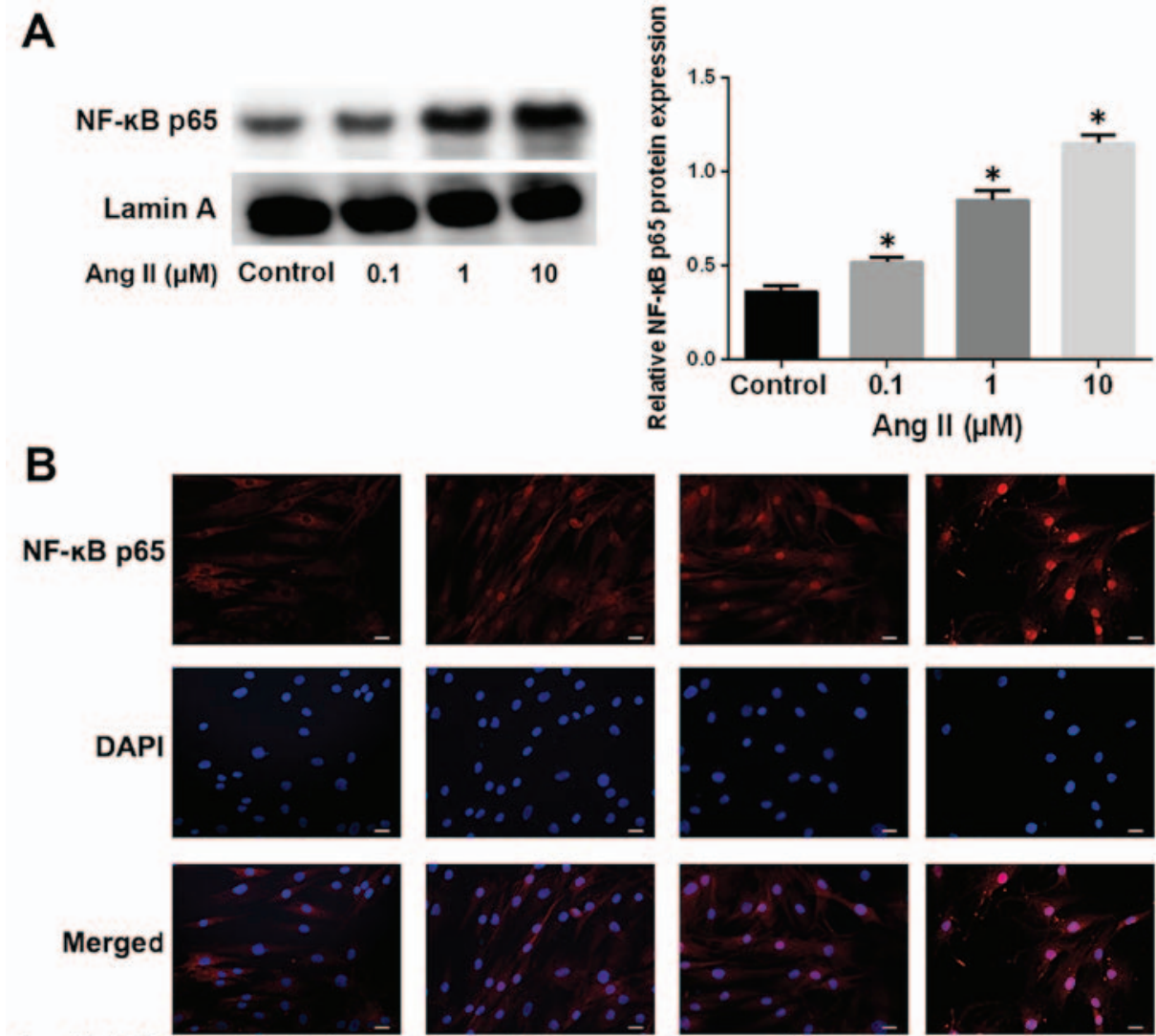

Ang II $(\mu \mathrm{M})$

Control

0.1

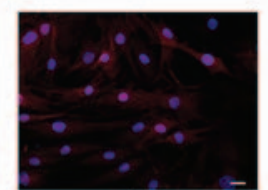

1

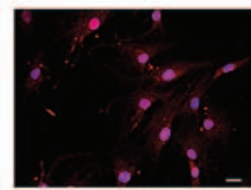

10

Figure 3. The effect of angiopoietin-2 (Ang-2) on nuclear translocation of NF-kB p65. Human nucleus pulposus (NP) cells were treated with various concentrations of exogenous Ang-2 (0.1, 1 and $10 \mu \mathrm{M})$ for $24 \mathrm{~h}$, with untreated cells serving as a control. (A) Protein levels of NF- $\mathrm{\kappa B}$ p65 in the nuclear fraction were determined by western blotting. Lamin A was used as an internal control. (B) Immunofluorescence staining of NF- $\kappa B$ p65 in treated NP cells. Scale bars, $20 \mu \mathrm{m}$. "P<0.05 vs. control group. 

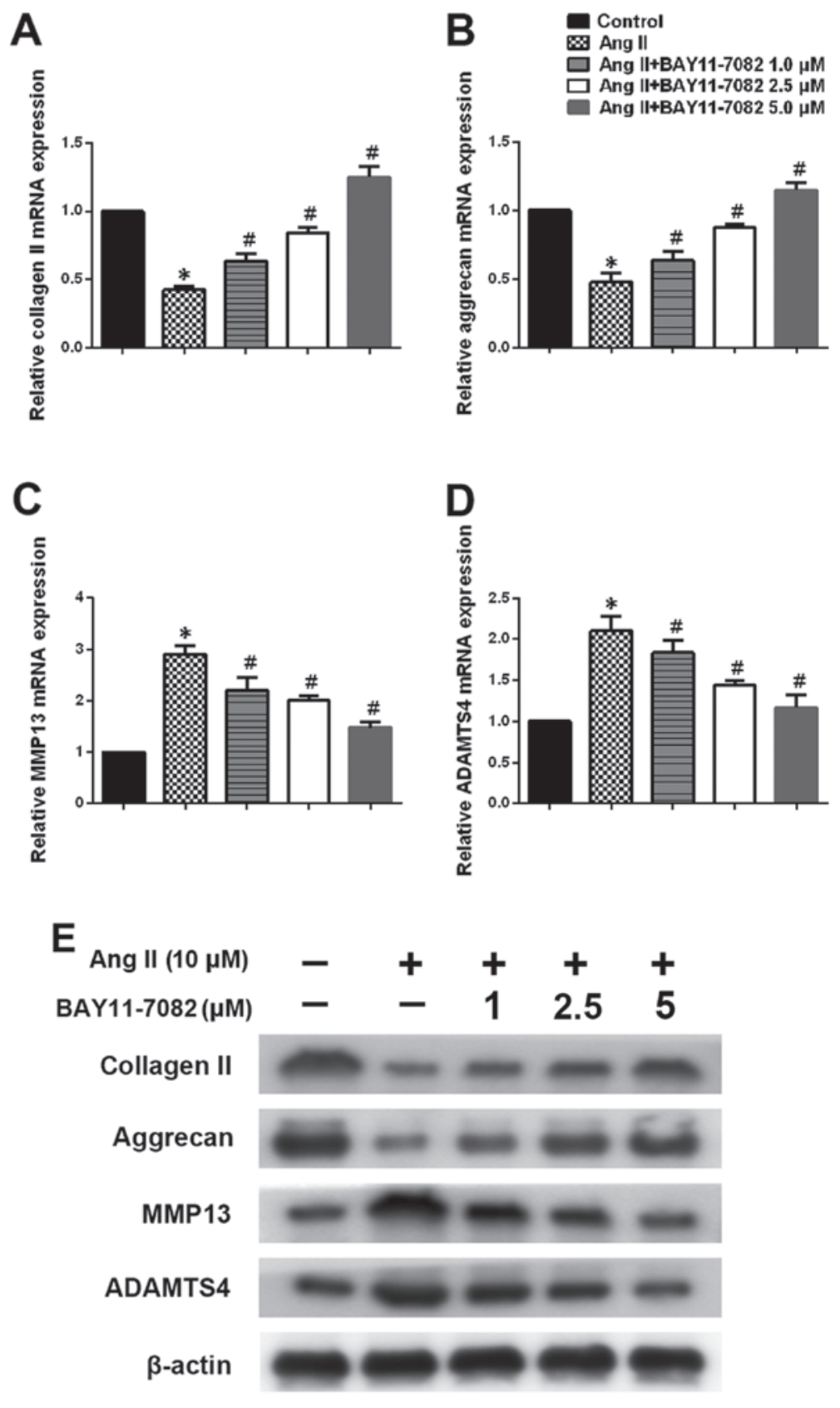

Figure 4. The NF-kB pathway mediates angiopoietin-2 (Ang-2)-induced extracellular matrix (ECM) degradation in nucleus pulposus (NP) cells. NP cells were treated with exogenous Ang-2 $(10 \mu \mathrm{M})$, in the presence or absence of various concentrations $(1,2.5$ and $5 \mu \mathrm{M})$ of the NF- $\mathrm{kB}$ inhibitor BAY11-7082. Untreated cells served as a control. (A) The expression of genes encoding type II collagen, (B) aggrecan, (C) MMP13 and (D) ADAMTS4 in NP cells was assessed by quantitative real-time polymerase chain reaction (qRT-PCR). (E) Protein levels of type II collagen, aggrecan, MMP13 and ADAMTS4 in NP cells were determined by western blotting. $\beta$-actin was used as an internal control. ${ }^{*} \mathrm{P}<0.05$ vs. control group; ${ }^{*} \mathrm{P}<0.05$ vs. Ang-2-only stimulation group.

induction of NF- $\kappa$ B p65 nuclear translocation by Ang-2 was also verified by immunofluorescence staining (Fig. 3B).

The effect of Ang-2 on ECM degradation is mediated by $N F-\kappa B$ in human degenerative NP cells. We next determined the expression of type II collagen, aggrecan, MMP13 and ADAMTS4 in NP cells stimulated by Ang- 2 in the presence or absence of NF- $\mathrm{kB}$ inhibitor BAY11-7082. Ang-2 treatment led to a marked downregulation of type II collagen and aggrecan expression in NP cells, whereas NF- $\kappa B$ inhibition reversed this effect in a dose-dependent manner (Fig. 4A, B and E). We also assessed the expression of MMP13 and ADAMTS4 on mRNA and protein levels. Upregulation of MMP13 and ADAMTS4 on stimulation with Ang-2 was significantly attenuated in the presence of the NF-kB inhibitor (Fig. 4C-E).

Ang-2 affects the expression of IL-1 $\beta$ in human degenerative $N P$ cells. The potent pro-inflammatory cytokine IL-1 $\beta$ plays an important role in the pathogenesis of IDD by hampering matrix synthesis and promoting the production of degradative enzymes (22). Ang-2 treatment significantly upregulated IL-1 $\beta$ mRNA and protein levels in NP cells a dose- and time-dependent manner (Fig. 5A-D). Furthermore, this effect was significantly attenuated in the presence of the NF- $\mathrm{KB}$ 

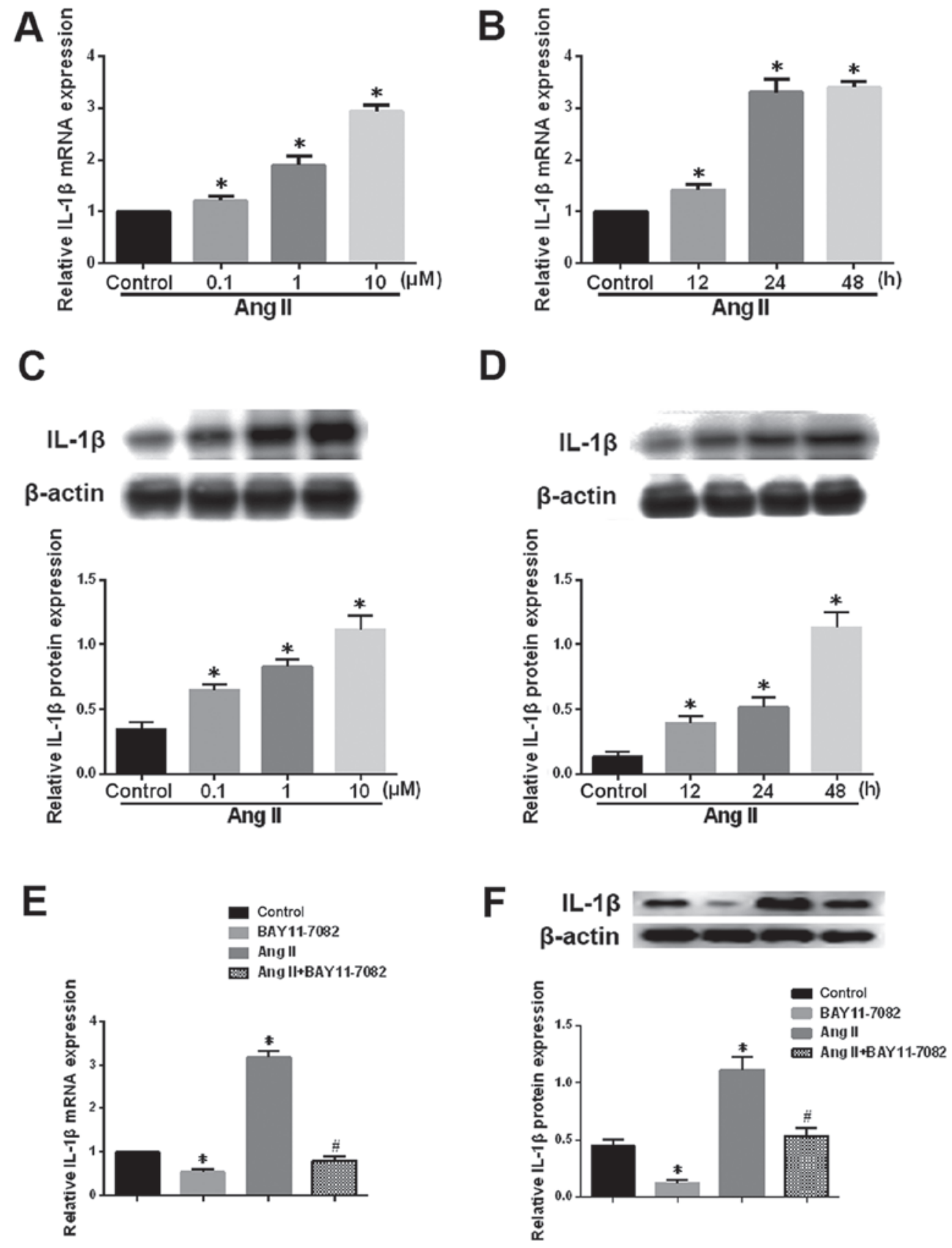

Figure 5. Effects of angiopoietin-2 (Ang-2) on interleukin (IL)-1 $\beta$ expression in human nucleus pulposus (NP) cells. (A-D) NP cells were treated with various concentrations $(0.1,1$ and $10 \mu \mathrm{M})$ of exogenous Ang-2 for $24 \mathrm{~h}$ or with $10 \mu \mathrm{M}$ of Ang-2 for different periods of time (12, 24 and $48 \mathrm{~h})$, with untreated cells serving as a control. (A and B) The mRNA and (C and D) protein levels of IL-1 $\beta$ were analyzed by quantitative real-time polymerase chain reaction (qRT-PCR) and western blotting, respectively. $\beta$-actin was used as an internal control. ${ }^{*} \mathrm{P}<0.05$ vs. control group. (E and F) NP cells were pretreated with the NF- $\kappa \mathrm{B}$ inhibitor BAY11-7082 $(5 \mu \mathrm{M})$ for $2 \mathrm{~h}$ and then treated with Ang-2 $(10 \mu \mathrm{M})$ for $24 \mathrm{~h}$. Untreated cells served as a control. (E) The IL-1 $\beta$ mRNA and (F) protein levels were assessed by qRT-PCR and western blotting, respectively. $\beta$-actin was used as an internal control. ${ }^{*} \mathrm{P}<0.05$ vs. control group; ${ }^{*} \mathrm{P}<0.05$ vs. Ang-2-only stimulation group.

inhibitor (Fig. 5E and F). These results suggest that the NF- $\mathrm{\kappa B}$ pathway mediates the Ang-2-induced expression of IL-1 $\beta$ in human NP cells.

\section{Discussion}

Accumulating evidence implicates altered cell function and decrease in the overall number of matrix-producing cells as potential contributors to IDD (23). Therefore, the control of matrix homeostasis, i.e., synthesis and degradation, is important. Numerous studies have focused on the Ang-2 epigenetic regulation of pathogenesis and potential Ang-2 targets in the tumor, specifically Ang/Tie signaling; however, the role of Ang-2 in IDD remains largely unknown. In the present study, we show that increased secretion of Ang-2 by NP cells is involved in IDD. By employing loss-of-function and gain-of-function approaches, we determined that Ang-2 plays a role in the degradation of ECM. Ang-2 promoted the activation of MMPs and ADAMTSs through the NF- $\kappa B$ signaling pathway. Inhibition of NF- $\kappa \mathrm{B}$ significantly suppressed this 
effect. Our data additionally indicated that Ang2 stimulates the expression of the pro-inflammatory cytokine IL-1 $\beta$ in human degenerative NP cells.

While gene expression profiling studies have identified endothelial cells as the primary source of Ang-2 (24), other studies have revealed that Ang-1 and -2 are also located at sites of endochondral bone formation in the growing skeleton (25). Mesenchymal stem cells, which express Ang-1 and high levels of Ang-2, possess a pronounced ability to elicit remodeling of preexisting vasculature at a site of injury and support new vessel formation (26). In the present study, examination of Ang-2 transcript and protein levels in NP cells derived from mild IDD and severe IDD confirmed that the expression of Ang-2 considerably increases during IDD progression. In addition, immunohistochemistry experiments revealed higher numbers of Ang-2 and MMP13-positive cells, and lower numbers of collage II-positive cells, in severe IDD than in mild IDD.

Accumulating evidence suggests that neovascularization of IVD is a pathological phenomenon as normal (healthy) discs are primarily avascular structures. A recent study evaluated the effect of extracellular endothelial microparticles (EMPs) and soluble protein factors (SUP fraction) produced by ECs on matrix catabolismin annulus fibrosus (AF) cells. This study revealed enhanced matrix catabolism as a molecular consequence of AF exposure to ECEMPs (27). Another study showed that AF cells from degenerated discs secrete factors that stimulate ECs to produce factors known to induce matrix degradation, angiogenesis and innervation (28). In the present study, we investigated the effect of Ang-2 on the expression of MMP13, ADAMTS4, aggrecan and collagen II. Gene expression analysis revealed that MMP13 and ADAMTS4 mRNA levels were significantly higher in the Ang-2 treatment group than in the control group. The effect of Ang-2 on aggrecan and type II collagen showed the opposite trend. Similarly, it has been shown that the treatment of cultured retinal microvascular endothelial cells with increasing amounts of purified Ang-2 led to a significant increase in MMP9 levels during retinal neovascularization (29).

In addition to the Ang/Tie system, numerous studies have focused on novel signaling pathways in non-Tie-expressing cells. Ang-2 has been shown to induce pericyte apoptosis via the p53 pathway under high glucose in diabetic retinopathy (30). Ang-2 addtionally exerts synergistic effects under high glucose conditions on astrocyte apoptosis via the GSK-3 $\beta / \beta$-catenin pathway (31). In another study, Ang-2 acts as a vessel-destabilizing molecule in TIE2-expressing ECs, and additionally as a direct proangiogenic molecule in TIE2-negative angiogenic ECs via integrin signaling (32). Moreover, Ang-2 interacts directly with integrins and activates the downstream focal adhesion kinase (FAK), ILK, Akt and ERK signaling pathways in Tie2-deficent glioma and breast cancer cells (33).

As a rapidly inducible transcription factor, $\mathrm{NF}-\kappa \mathrm{B}$ regulates the expression of numerous genes to mediate various cellular processes, including cell proliferation, survival and differentiation (34). Furthermore, NF- $\mathrm{BB}$ is an important pathway that plays a crucial role in both anabolism and catabolism in IVD. The decreased gene expression of aggrecan and type II collagen induced by IL-1 $\beta$ was reversed by inhibiting NF- $\mathrm{NB}$ signaling (35). NF- $\kappa \mathrm{B}$ pathway promotes catabolic gene expression such as MMP-1, MMP-3, MMP-13, ADAMTS-4 and ADAMTS-5 expression in IL-1 $\beta$ stimulated animal NP cells (36). Here, we mainly studied the NF- $\kappa \mathrm{B}$ signaling pathway in Ang-2 treated degenerative NP cells. The results showed that the effect of Ang-2 on ECM degradation is mediated by NF- $\kappa \mathrm{B}$ signaling pathway, at least in part. When we used an inhibitor of NF- $\mathrm{NB}$, the effect of Ang-2 on ECM degradation was attenuated. Catabolism relative ADAMTS4 and MMP13 expression induced by Ang-2 decreased dramatically. However, increased synthesis collagen II and aggrecan were detected after BAY117082 treatment. Future studies should investigate the specific mechanism whereby Ang- 2 activates $\mathrm{NF}-\kappa \mathrm{B}$ signaling in degenerative NP cells. In addition, Ang-2 induces the expression of IL-1 $\beta$ in a time- and concentration-dependent manner in human degenerative NP cells. This process was additionally weaken by the inhibitor of $\mathrm{NF}-\kappa \mathrm{B}$.

David et al observed that expression of neovascularization growth factors in the disc is associated with post-surgical pain, implicating this process in a clinically relevant pathology (37). A phase III AVAGAST trial is currently under way to evaluate Ang-2 as a biomarker in gastric cancer (38). To the best of our knowledge, this study provides evidence for the first time that Ang-2 levels in degenerative NP cells increase with increasing severity of IDD. Upon Ang-2 exposure, MMPs and ADAMTSs become dysregulated in degenerative NP cells and degrade aggrecan and type II collagen. This contributes to the pathogenesis of IDD. Ang-2 is also involved in the pathogenesis of IDD, at least partially, by inducing $\mathrm{NF}-\kappa \mathrm{B}$ activation and IL-1 $\beta$ expression.

In conclusion, our study suggests that the inhibition of Ang-2 may represent a novel therapeutic approach for the treatment of IDD.

\section{Acknowledgements}

Not applicable.

\section{Funding}

This study was supported by the National Natural Science Foundation of China (nos. 81272025 and 81541056).

\section{Availability of data and material}

The datasets used and/or analyzed during the current study are available from the corresponding author on reasonable request.

\section{Authors' contributions}

CY designed the study. CY and KW wrote the manuscript. $\mathrm{KW}, \mathrm{LK}$, and WL conducted the experiments. YS, XW, YZ, WH, KZ, SL, LT and RL collected the data. YS, XW, and YZ analyzed the data. CY reviewed and revised the manuscript.

\section{Ethics approval and consent to participate}

This study was approved by the Institutional Ethics Review Board of Tongji Medical College of Huazhong University of Science and Technology.

\section{Consent for publication}

Not applicable. 


\section{Competing interests}

The authors declare that they have no competing interests.

\section{References}

1. Deyo RA and Weinstein JN: Low back pain. N Engl J Med 344: 363-370, 2001.

2. Sakai D and Andersson GB: Stem cell therapy for intervertebral disc regeneration: obstacles and solutions. Nat Rev Rheumatol 11: 243-256, 2015

3. Kepler CK, Ponnappan RK, Tannoury CA, Risbud MV and Anderson DG: The molecular basis of intervertebral disc degeneration. Spine J 13: 318-330, 2013.

4. Phillips KL, Jordan-Mahy N, Nicklin MJ and Le Maitre CL: Interleukin-1 receptor antagonist deficient mice provide insights into pathogenesis of human intervertebral disc degeneration. Ann Rheum Dis 72: 1860-1867, 2013.

5. Le Maitre CL, Freemont AJ and Hoyland JA: Localization of degradative enzymes and their inhibitors in the degenerate human intervertebral disc. J Pathol 204: 47-54, 2004.

6. Bachmeier BE, Nerlich A, Mittermaier N, Weiler C, Lumenta C, Wuertz K and Boos N: Matrix metalloproteinase expression levels suggest distinct enzyme roles during lumbar disc herniation and degeneration. Eur Spine J 18: 1573-1586, 2009.

7. Makinde TO and Agrawal DK: Increased expression of angiopoietins and Tie2 in the lungs of chronic asthmatic mice. Am J Respir Cell Mol Biol 44: 384-393, 2011.

8. Kim H and Koh GY: Ang2, the instigator of inflammation. Blood 118: 4767-4768, 2011.

9. Scholz A, Lang V, Henschler R, Czabanka M, Vajkoczy P, Chavakis E, Drynski J, Harter PN, Mittelbronn M, Dumont DJ, et al: Angiopoietin-2 promotes myeloid cell infiltration in a $\beta_{2}$-integrin-dependent manner. Blood 118 : 5050-5059, 2011.

10. Fiedler U, Reiss Y, Scharpfenecker M, Grunow V, Koidl S, Thurston G, Gale NW, Witzenrath M, Rosseau S, Suttorp N, et al Angiopoietin-2 sensitizes endothelial cells to TNF-alpha and has a crucial role in the induction of inflammation. Nat Med 12: 235-239, 2006

11. Fiedler U and Augustin HG: Angiopoietins: a link between angiogenesis and inflammation. Trends Immunol 27: 552-558, 2006.

12. Tabruyn SP, Colton K, Morisada T, Fuxe J, Wiegand SJ, Thurston G, Coyle AJ, Connor J and McDonald DM: Angiopoietin-2-driven vascular remodeling in airway inflammation. Am J Pathol 177: 3233-3243, 2010.

13. Setton LA and Chen J: Mechanobiology of the intervertebral disc and relevance to disc degeneration. J Bone Joint Surg Am 88 (Suppl 2): 52-57, 2006.

14. Sakai D, Nakamura Y, Nakai T, Mishima T, Kato S, Grad S, Alini M, Risbud MV, Chan D, Cheah KS, et al: Exhaustion of nucleus pulposus progenitor cells with ageing and degeneration of the intervertebral disc. Nat Commun 3: 1264, 2012.

15. Thompson JP, Pearce RH, Schechter MT, Adams ME, Tsang IK and Bishop PB: Preliminary evaluation of a scheme for grading the gross morphology of the human intervertebral disc. Spine 15: 411-415, 1990

16. Pfirrmann CW, Metzdorf A, Zanetti M, Hodler J and Boos N: Magnetic resonance classification of lumbar intervertebral disc degeneration. Spine 26: 1873-1878, 2001.

17. Liu W, Zhang Y, Feng X, Li S, Gao Y, Wang K, Song Y, Yang S, Tu J, Shao Z, et al: Inhibition of microRNA-34a prevents IL-1 $\beta$-induced extracellular matrix degradation in nucleus pulposus by increasing GDF5 expression. Exp Biol Med (Maywood) 241: 1924-1932, 2016.

18. Wu X, Liu W, Duan Z, Gao Y, Li S, Wang K, Song Y, Shao Z, Yang S and Yang C: The involvement of protease nexin-1 (PN1) in the pathogenesis of intervertebral disc (IVD) degeneration. Sci Rep 6: 30563, 2016.

19. Pockert AJ, Richardson SM, Le Maitre CL, Lyon M, Deakin JA, Buttle DJ, Freemont AJ and Hoyland JA: Modified expression of the ADAMTS enzymes and tissue inhibitor of metalloproteinases 3 during human intervertebral disc degeneration. Arthritis Rheum 60: 482-491, 2009.

20. Tian Y, Yuan W, Fujita N, Wang J, Wang H, Shapiro IM and Risbud MV: Inflammatory cytokines associated with degenerative disc disease control aggrecanase-1 (ADAMTS-4) expression in nucleus pulposus cells through MAPK and NF- $\kappa \mathrm{B}$. Am J Pathol 182: 2310-2321, 2013.
21. Wang X, Wang H, Yang H,Li J, CaiQ, Shapiro IM and Risbud MV: Tumor necrosis factor- $\alpha$ - and interleukin-1 $\beta$-dependent matrix metalloproteinase-3 expression in nucleus pulposus cells requires cooperative signaling via syndecan 4 and mitogen-activated protein kinase-NF- $\mathrm{B}$ axis: implications in inflammatory disc disease. Am J Pathol 184: 2560-2572, 2014.

22. Phillips KL, Cullen K, Chiverton N, Michael AL, Cole AA, Breakwell LM, Haddock G, Bunning RA, Cross AK and Le Maitre CL: Potential roles of cytokines and chemokines in human intervertebral disc degeneration: interleukin-1 is a master regulator of catabolic processes. Osteoarthritis Cartilage 23: 1165-1177, 2015.

23. Roughley PJ: Biology of intervertebral disc aging and degeneration: involvement of the extracellular matrix. Spine 29: 2691-2699, 2004.

24. Fiedler U, Scharpfenecker M, Koidl S, Hegen A, Grunow V, Schmidt JM, Kriz W, Thurston G and Augustin HG: The Tie-2 ligand angiopoietin- 2 is stored in and rapidly released upon stimulation from endothelial cell Weibel-Palade bodies. Blood 103: 4150-4156, 2004.

25. Horner A, Bord S, Kelsall AW, Coleman N and Compston JE: Tie2 ligands angiopoietin-1 and angiopoietin-2 are coexpressed with vascular endothelial cell growth factor in growing human bone. Bone 28: 65-71, 2001.

26. Nagano M, Kimura K, Yamashita T, Ohneda K, Nozawa D, Hamada H, Yoshikawa H, Ochiai N and Ohneda O: Hypoxia responsive mesenchymal stem cells derived from human umbilical cord blood are effective for bone repair. Stem Cells Dev 19: 1195-1210, 2010.

27. Pohl PH, Lozito TP, Cuperman T, Yurube T, Moon HJ, Ngo K, Tuan RS, St Croix C, Sowa GA, Rodrigues LM, et al: Catabolic effects of endothelial cell-derived microparticles on disc cells: implications in intervertebral disc neovascularization and degeneration. J Orthop Res 34: 1466-1474, 2016.

28. Moon HJ, Yurube T, Lozito TP, Pohl P, Hartman RA, Sowa GA, Kang JD and Vo NV: Effects of secreted factors in culture medium of annulus fibrosus cells on microvascular endothelial cells: elucidating the possible pathomechanisms of matrix degradation and nerve in-growth in disc degeneration. Osteoarthritis Cartilage 22: 344-354, 2014.

29. Das A, Fanslow W, Cerretti D, Warren E, Talarico N and McGuire P: Angiopoietin/Tek interactions regulate $\mathrm{mmp}-9$ expression and retinal neovascularization. Lab Invest 83: 1637-1645, 2003

30. Park SW, Yun JH, Kim JH, Kim KW, Cho CH and Kim JH: Angiopoietin 2 induces pericyte apoptosis via $\alpha 3 \beta 1$ integrin signaling in diabetic retinopathy. Diabetes 63: 3057-3068, 2014.

31. Yun JH, Park SW, Kim JH, Park YJ, Cho CH and Kim JH: Angiopoietin 2 induces astrocyte apoptosis via $\alpha v \beta 5$-integrin signaling in diabetic retinopathy. Cell Death Dis 7: e2101, 2016.

32. Felcht M, Luck R, Schering A, Seidel P, Srivastava K, Hu J, Bartol A, Kienast Y, Vettel C, Loos EK, et al: Angiopoietin-2 differentially regulates angiogenesis through TIE2 and integrin signaling. J Clin Invest 122: 1991-2005, 2012

33. Lee HS, Oh SJ, Lee KH, Lee YS, Ko E, Kim KE, Kim HC, Kim S, Song PH, Kim YI, et al: Gln-362 of angiopoietin-2 mediates migration of tumor and endothelial cells through association with $\alpha 5 \beta 1$ integrin. J Biol Chem 289: 31330-31340, 2014.

34. Hayden MS and Ghosh S: NF- $\kappa B$, the first quarter-century: remarkable progress and outstanding questions. Genes Dev 26: 203-234, 2012.

35. Zhongyi S, Sai Z, Chao L and Jiwei T: Effects of nuclear factor kappa B signaling pathway in human intervertebral disc degeneration. Spine 40: 224-232, 2015.

36. Ellman MB, Kim JS, An HS, Kroin JS, Li X, Chen D, Yan D, Buechter DD, Nakayama K, Liu B, et al: The pathophysiologic role of the protein kinase C $\delta$ pathway in the intervertebral discs of rabbits and mice: in vitro, ex vivo, and in vivo studies. Arthritis Rheum 64: 1950-1959, 2012.

37. David G, Ciurea AV, Iencean SM and Mohan A: Angiogenesis in the degeneration of the lumbar intervertebral disc. J Med Life 3: 154-161, 2010.

38. Hacker UT, Escalona-Espinosa L, Consalvo N, Goede V, Schiffmann L, Scherer SJ, Hedge P, Van Cutsem E, Coutelle O and Büning H: Evaluation of angiopoietin-2 as a biomarker in gastric cancer: results from the randomised phase III AVAGAST trial. Br J Cancer 114: 855-862, 2016. 